\title{
Platelet Aggregation Increased by Advanced Glycated Hemoglobin
}

Asadifar $\mathbf{M}^{1}$, Bakhti $\mathbf{M}^{1}$, Habibi-Rezaei $\mathbf{M}^{1^{*}}$, Moosavi-Movahedi $\mathrm{AA}^{2}$, Tabatabi $\mathbf{M R}^{3}$, Ahmadinejad $\mathbf{M}^{3}$ and Badlou $\mathrm{BA}^{2^{*}}$

${ }^{1}$ School of Biology, College of Science, University of Tehran, Tehran, Iran

${ }^{2}$ Institute of Biochemistry and Biophysics, University of Tehran, Tehran, Iran

${ }^{3}$ Blood Transfusion Research Center, High Institute for Research and Education, Iranian blood bank Organization, IBTO, Tehran, Iran

${ }^{4}$ BBAdvies and research, Research and Development Department, Zeist, The Netherlands

"Corresponding authors: Bahram Alamdary Badlou, BBAdvies and Research, Research and Development Dept. Zeist, The Netherlands, Tel: +31302211328; Fax: +31302211328; E-mail: bbadlou@casema.nl

Habibi-Rezaei M, School of Biology, College of Science, University of Tehran, P.O. Box 14155-6455, Tehran, Iran, Tel: +98-21-61113214; Fax: +98-21-6640514; E-mail: mhabibi@khayam.ut.ac.ir

Received date: May 20, 2015, Accepted date: July 30, 2015, Publication date: Aug 03, 2015

Copyright: (C) 2015 Asadifar M, et al. This is an open-access article distributed under the terms of the Creative Commons Attribution License, which permits unrestricted use, distribution, and reproduction in any medium, provided the original author and source are credited.

\begin{abstract}
Introduction: Many studies have provided strong evidence for an association between diabetes complications and an increase in platelet (PLT) reactivity. Though some metabolic abnormalities have been reported as the major causes of this reactivity and malfunction, the precise mechanism has not been fully elucidated.
\end{abstract}

Aim: The aim of this study is to investigate the effect of advanced glycation end product of hemoglubin (AGE-Hb) on human PLT reactivity and malfunction.

Materials and Methods: A solution of bovine hemoglobin was prepared with fructose in phosphate buffer. The solution was then sterilized and incubated under sterile conditions at $37^{\circ} \mathrm{C}$ in the dark. The control solution was prepared in the same way, but without fructose. Human PLTs were isolated and prepared from 15 healthy volunteers, men and women. Blood was collected in the morning from fasting healthy subjects using a 21-gauge needle with $117 \mathrm{mM}$ sodium citrate $(1: 9 \mathrm{v} / \mathrm{v})$, as an anticoagulant. Fluorescence measurements were performed using a Hitachi F-4500 spectrofluorometer. PLT aggregation was measured using the photometric system Packs-4 aggregometer.

Results: The relative fluorescence intensity increased in $\mathrm{Hb}$ samples but not in controls that incubated with fructose. PLTS aggregation did not change in controls while after incubation with 10, 22, 30-days fructose-glycated $\mathrm{Hb}$ decreased by $10,12,30 \%$, respectively.

Taken together our data show that PLT secondary phase of ADP-induced aggregation gradually affected with advanced increase in $\mathrm{Hb}$ glycation.

Keywords: Human platelets; Diabetes; HbAc; Glycation; Blood transfusion; Hyperreactivity; In-vitro

\section{Abbreviations:}

PLTS: Human Blood Platelets; AGE: Advanced Glycation End Products; AGE-BSA: Advanced Glycation End Product-BSA; AGEHb: Advanced Glycation End Products-Hemoglobin

\section{Introduction}

Platelets (PLTs), the smallest enucleated cells in blood stream derived from bone marrow megakaryocytes, play some critical roles in haemostasis, thrombosis, and atherosclerosis [1]. It has been reported that PLTs from diabetic patients have different properties with those from normal individuals [2]. Many studies have also provided strong evidence for an association between diabetic complications and increase in PLT function defect and hyperactivity [3]. Though some metabolic abnormalities in proteins extracellular and/or interacelluarly have been reported as the major causes of this hyperactivity, the precise mechanism has not been fully elucidated $[4,5]$.

However, it has been shown that free radicals increase the aggregation of PLTs5. Since these molecules are the major by-products of glycation reactions [6], they have been speculated as an important cause of PLT function inadequacy in diabetes.

Non-enzymatic glycosylation or glycation is a process in which reducing sugar(s) react spontaneously with amino groups in proteins to produce Advanced Glycation End products (AGEs) [7]. This phenomenon introduces considerable alteration on the structure of proteins, and consequently changes their function [8]. The chemistry of AGEs is not fully understood; in the meantime increased levels of circulating AGEs have been demonstrated in both animal and human diabetics [9]. AGE structures are associated with many pathophysiological abnormalities [10] as in diabetes and aging [11,12].

It has been assumed that PLT hyperactivity is influenced by protein glycation upon different mechanisms i.e. glycation of PLT membrane proteins [13], glycation of PLT cytosolic proteins [14], and interaction 
between glycated proteins or AGEs and PLTs [15-17]. Reports dealing with the interaction between AGEs and PLTs are limited to few cases in leterature. Hangaishi et al. have reported an increase in PLT aggregation upon treatment with AGE-BSA [16]. Furthermore, [17] Ferreti et al. have observed the same phenomenon during incubation of PLTs with glycated LDL, [17] along with some changes in PLT metabolism.

The most known form of the glycation product of hemoglobin is $\mathrm{HbA1c}$, which has been attained significant prominence in the modern world of medical biology due to its use as a scale in the long-term control of diabetes mellitus $[9,18,19]$ and aging-accelerated glycation diseases $[11,18]$. HbAlc is formed through a non enzymatic reaction between glucose and $\mathrm{N}$-terminal valin residue of the $\beta$-chain of $\mathrm{HbA0}$ [20].

In vivo, advancement of the glycation process, mostly involved by lysyl $\varepsilon$-amino groups, results in advanced glycated end products of $\mathrm{Hb}$ (AGE-Hb). These structures have been reported with vigorous altered structural features [21]. However, the presence and circulation of $0.42 \%$ and $0.75 \%$ AGE-Hb have been reported in non-diabetic and diabetic patients, respectively [19]. Regarding to the higher plasma concentration of AGE-Hb in diabetic patients $[19,21]$, the interaction between this molecule and other blood elements such as PLTs in diabetics may changes the PLTs (ir)responsiveness and possible premature agglutionations, forming of non firmed revesible aggregates.

The aim of this study is to investigate the effect of AGE-Hb on human PLT aggregation, invitro.

Here we show that PLT secondary phase of ADP-induced aggregation affected by gradual increase in Hbglycation, as it is previously reported with increased glycated LDL17- and AGE-BSA [18].

\section{Material}

Bovine methemoglobin, and Griess reagent were obtained from Sigma Chemical Co. (St. Louis, Mo, USA). Thioflavin T was purchased from Fluka/Sigma-Aldrich (St.Louis, USA). All other chemicals were purchased from Merck (Darmstadt, Germany).

\section{Blood sample preparation}

This study was performed on 15 healthy volunteers, men and women, aged 22 to 40 years. Blood was collected in the morning from fasted healthy subjects using a 21-gauge needle with $117 \mathrm{mM}$ sodium citrate $(1: 9 \mathrm{v} / \mathrm{v})$, as an anticoagulant. None of the subjects had any signs or history of serious diseases, and they had abstained from taking medication for at least 10 days. Oral informed consent was obtained from all volunteers.

Whole blood was centrifuged at $150 \times \mathrm{g}$ for 10 minutes at $25^{\circ} \mathrm{C}$ to prepare PLT-rich plasma (PRP), and the remaining supernatant was centrifuged at $2500 \times \mathrm{g}$ for 10 minutes at $25^{\circ} \mathrm{C}$ to obtain PLT-poor plasma (PPP). The PLTs count in PRP was measured and the PRP was adjusted to $3.8 \times 10^{5} / \mu \mathrm{l}$ with PPP.

\section{Invitro preparation of AGE-Hb}

A solution of bovine hemoglobin $(15 \mu \mathrm{M})$ was prepared with 30 $\mathrm{mM}$ fructose in a $50 \mathrm{mM}$ phosphate buffer with $\mathrm{pH} 7.4$, containing $0.02 \%(\mathrm{~W} / \mathrm{V})$ sodium azide. The solution was sterilized by filtering through low protein binding filter (Millex. •.-GV 0.22. $\mu \mathrm{m}$ filter unit, Millipore, USA) and was then incubated under sterile conditions at $37^{\circ} \mathrm{C}$ in the dark.

The control solution was prepared in the same way, but without fructose. Samples were taken at various intervals and dialyzed extensively against $50 \mathrm{mM}$ phosphate buffer with $\mathrm{pH} 7.4$ at $4^{\circ} \mathrm{C}$, to remove unbounded fructose molecules. After dialysis protein concentrations were estimated by the Bradford method [22] and the samples were immediately frozen and stored at $-70^{\circ} \mathrm{C}$ until processed.

\section{AGE-Hb associated fluorescence analysis}

Fluorescence measurements were performed using a Hitachi F-4500 spectrofluorometer. AGE-related autofluorescence of the sample preparations was monitored by exciting at $370 \mathrm{~nm}$ and emission wavelength at $440 \mathrm{~nm}$ at $25^{\circ} \mathrm{C}$ which was determined through wavelength scanning in the range of $400-500 \mathrm{~nm}$. The spectra were corrected with appropriate protein and buffer blanks. For all measurements, the samples contained $0.1 \mathrm{mg} / \mathrm{ml}$ protein.

\section{Circular dichroism spectropolarimetry}

Circular dichroism (CD) spectra were collected between 190 and $260 \mathrm{~nm}$, at the far UV region with an Aviv CD spectropolarimeter model 215) at $25^{\circ} \mathrm{C}$. The $\mathrm{CD}$ measurement was performed using a $1 \mathrm{~mm}$ path length quartz cuvette, at the final protein concentration of $0.2 \mathrm{mg} / \mathrm{ml}$. Deconvolution of the data was performed to estimate the (a)-helix and ( $\beta$ )-conformation content of AGE-Hbs using CDNN software [23].

\section{Thioflavin T test}

Thioflavin $\mathrm{T}(\mathrm{ThT})$ is a benzothiazole dye that exhibits enhanced fluorescence upon binding to amyloid fibrils and is commonly used to diagnose amyloid fibrils, both exvivo and in vitro. Solutions of $1.5 \mu \mathrm{M}$ fructated hemoglobin and control samples were incubated at $25^{\circ} \mathrm{C}$ with $5 \mu \mathrm{M}$ thioflavin $\mathrm{T}$ for an hour. The free ThT remained in solution and did not affect signal-to-noise ratio. The sample fluorescence was measured through excitation at $435 \mathrm{~nm}$ and emission spectra were recorded in the wavelength range of $455-555 \mathrm{~nm}$; band slit was adjusted at $10 \mathrm{~nm}$.

\section{PLT aggregation}

PLT aggregation was measured using the photometric system Packs-4 aggregometer (Helena Laboratories, Beaumont, TX). In brief, $350 \mu \mathrm{l}$ of PRP obtained from subjects and $100 \mu \mathrm{l}$ of $3 \mu \mathrm{M}$ hemoglobin at various levels of glycation were incubated with stirring (1000rpm) for $15 \mathrm{~min}$ at $37^{\circ} \mathrm{C}$. PLTs count was adjusted to 300000 per microliter. Then $50 \mu \mathrm{ADP}(20 \mu \mathrm{M})$ was then added and the PLT aggregation was monitored for 15 minutes.

All aggregation phases were step-by-step analysed to unravel how glycated proteins affect agglutination and lag phase, shape change, primary phase, secondary phase, reversibility and maximal aggregation.

\section{NO measurement}

For NO assessment PLT aggregation was performed as described above. Fifteen minutes after PLT aggregation, $50 \mu \mathrm{l}$ of fixative solution (36 mM EDTA, 1\% formaldehyde) was added, and the samples were 
centrifuged at $12000 \mathrm{rpm}$ for $2 \mathrm{~min}$ at $4^{\circ} \mathrm{C}$. The production of NO by PLTs was assayed through measuring nitrite in the supernatant of centrifuged samples.

In brief, $100 \mu \mathrm{l}$ of supernatant of each sample was transferred to a 96-well flat-bottom microtiter plate and well mixed with equal volume of Griess reagent at room temperature for $10 \mathrm{~min}$. The absorbance values at $540 \mathrm{~nm}$ were measured on an EIA Multiscan MS microplate reader. Moreover, $\mathrm{NaNO}_{2}$ in a concentration range from 0 to $100 \mu \mathrm{M}$ was used to construct standard curve.

\section{Results}

\section{AGE-related fluorescence properties of $\mathrm{Hb}$}

The emitted fluorescence intensity values for fructated $\mathrm{Hb}$ and controls are plotted as a function of time in Figure 1. AGE-related fluorescence properties of the haemoglobin incubated with fructose were assessed through maximum emission peak at $440 \mathrm{~nm}$ after excitation at $370 \mathrm{~nm}$.

Fluorescence studies over a 30-day incubation period revealed an increase in the fluorescence intensity for the samples incubated with fructose along with incubation time. However, AGE-related increase in the intensity of fluorescence was not observed for the controls.

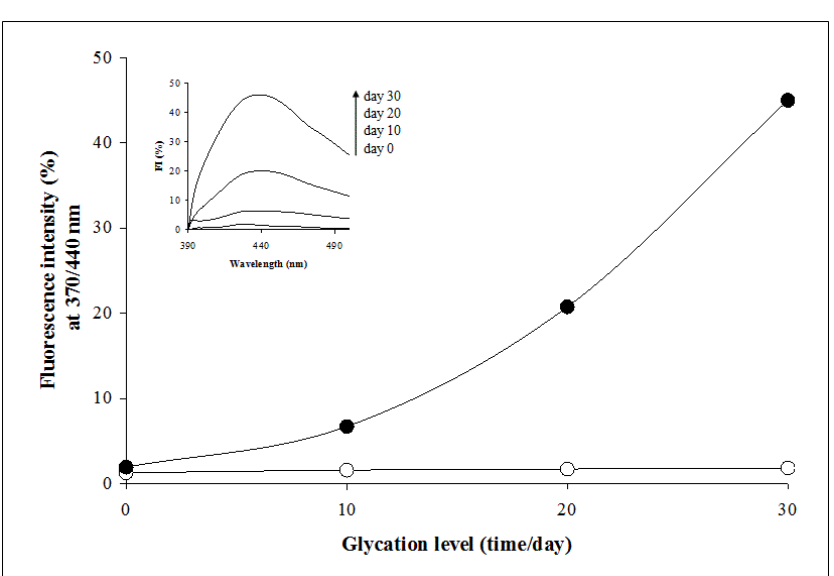

Figure 1: AGE-related fluorescence properties of $\mathrm{Hb}$. The emitted fluorescence intensity values for fructated $\mathrm{Hb}$ and controls are plotted as a function of time. Filled dots are AGE-Hb treated samples and empty dots are controls. In inset are shown AGErelated fluorescence properties of the haemoglobin incubated with fructose which were assessed through maximum emission peak at $440 \mathrm{~nm}$ after excitation at $370 \mathrm{~nm}$.

\section{The CD spectra at the far-UV region of AGE-Hb}

The CD spectra at the far-UV region of AGE-Hb are different from the controls and a significant change in the secondary structure of AGE-Hb was observed by a decrease in negative ellipticity in the wavelength rang of 205-235 nm (Figure 2).

Furthermore, in progressive glycation with fructose a decrease in $\alpha$ helicity along with an increase in $\beta$-conformation content in AGE-Hb was observed (data not shown).

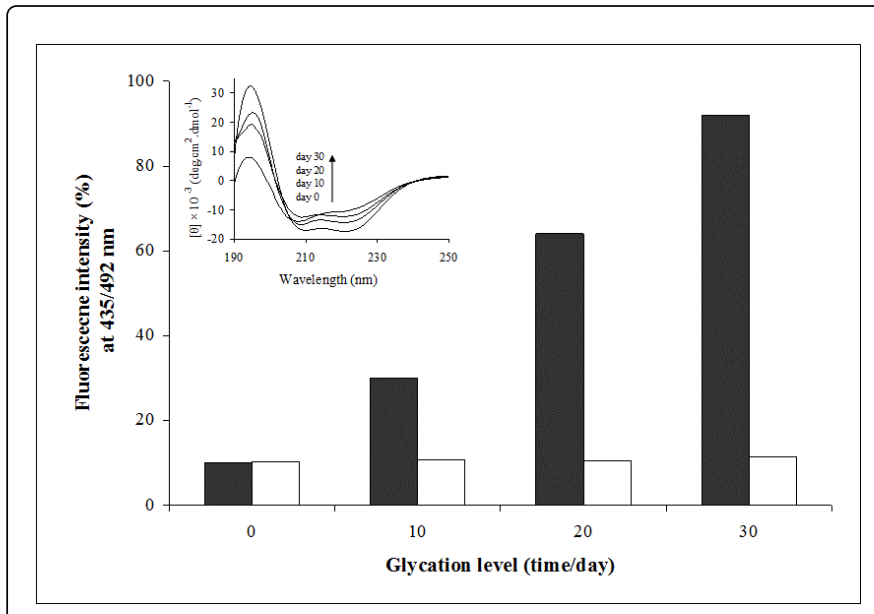

Figure 2: The CD spectra at the far-UV region of AGE-Hb. ThTbinding function against fructation time effect in which maximum fluorescence emission was measured through excitation at $435 \mathrm{~nm}$ and spectra were recoded in the wavelength range of $455-555 \mathrm{~nm}$ for glycated $\mathrm{Hb}(\square)$ and control samples ( $\square$ ). Inset to the figure presents resulting far-ultra-violet $\mathrm{CD}$ spectra for the samples of $\mathrm{Hb}$ treated with fructose during 30 days incubation time.

\section{The thioflavin T-related fluorescence intensity increased with increasing incubation time}

Fructated $\mathrm{Hb}$ was exposed to $\mathrm{ThT}$, and fluorescence emission was measured in different days. The ThT-related fluorescence intensity increased with increasing the time of incubation, which shows the progressive formation of the amyloid structures (Figure 2).

In the samples, a gradual increase in the fluorescence emission of thioflavin $\mathrm{T}$ was observed at $492 \mathrm{~nm}$ upon binding to the glycated protein but not in control solutions.

\section{AGE-Hb affects ADP-induced aggregation function}

After validation of AGE-Hb quantity and quality under in-vitro condition the effect of AGE-Hb on human PLT aggregation function was investigated. Generally, glycated hemoglobin was revealed to have a significant effect on ADP-induced aggregation of human PLTs invitro.

As it is shown in Figure 3 with only ADP preincubated PLTs showed no remarkable decrease in niether primary nor secondary phase of ADP-induced aggregation (control group; PRP+ADP curve); while preincubation of PLTs with AGE-Hbday0 for $15 \mathrm{~min}$, delayed and decreased the sceondary phase and maximal aggregation (PRP $+\mathrm{ADP}+\mathrm{AGEHb}$ day0).

PLT aggregation induced by ADP was significantly blocked and decreased along with increase in hemoglobin glycation incubation time day 22 and 30 as a function of time. However, control samples prepared in different days caused no significant changes in ADPinduced aggregation.

In all samples that exposed to ADP immidiately their primary phase aggregation were not affected by AGEHbs irrespective of AGEHbs incubation time but the secondary phase were specially affected, which needs more investigation (Figure 3 ). 


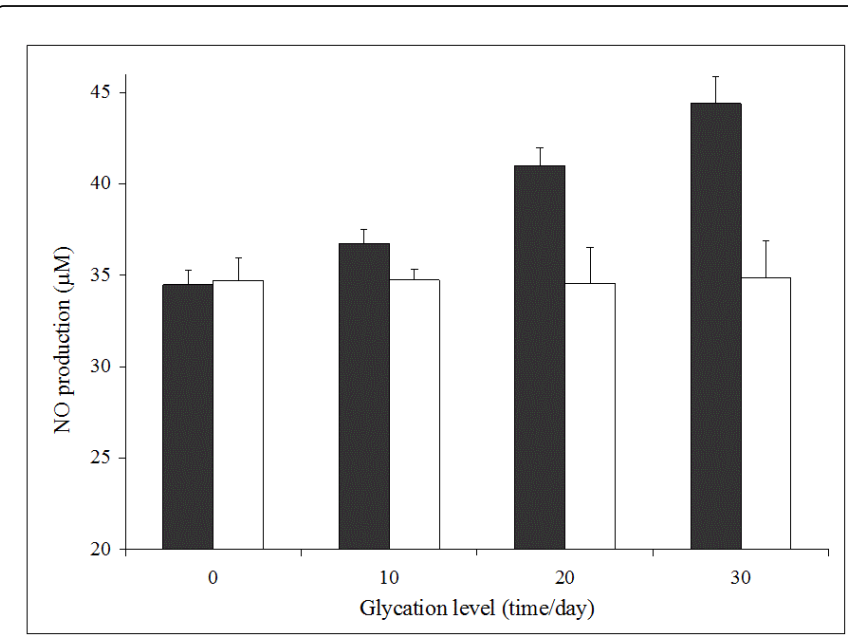

Figure 3: AGE-Hb caused remarkable increase in NO production. NO production in platelets pre-incubated with different level of glycated hemoglobin ( $)$ and control samples $(\square)$. Each value represents the mean \pm S.D. $(n=5)$.

\section{AGE-Hb caused significant increase in NO production}

AGE-Hb caused significant increase in NO production in aggregated PLTs induced by ADP, which was monitored through incubation time (Figure 3). The NO production in those cells treated with control samples, however, was negligible. This increase in NO production seems to be a consequence of more platelet aggregation in samples treated with $\mathrm{Hb}$-AGE.

\section{Discussion}

There are a lot of evidences which have shown a direct correlation between diabetic complications and PLTs reactivity [2]. This PLT malfunction has been attributed in part to hyperglycemia and glycation reactions that might aggravate with a higher rate in hyperglycemia conditions [24]. Aforementioned reactions affect PLT function by different mechanisms [15]. Several studies have revealed that an increase in PLT reactivity upon treatment with AGE proteins; though their influences vary remarkably [15-17].

In diabetics the AGE-Hb content raises extremely. Haemoglobin glycation results in an early and advanced glycation end-products, which are known as $\mathrm{HbAlc}$ and $\mathrm{AGE}-\mathrm{Hb}$, respectively. In our previous study we have shown that prolonged incubation of $\mathrm{Hb}$ with fructose increases consequential alteration in Hb structure [21]. In this study we show that pretreatment of PLTs with AGE-Hb affects ADPinduced PLT aggregation at different phases. These effects are absent in the controls and fresh proteins, which confirms previous studies dealing with the effects of AGE structures on these cells.

The general mechanism by which AGE proteins influence different cell types has been described to be involved particular types of receptors such as RAGE (receptor for AGE) and SR (scavenger receptor) [15,25].

Hasegawa et al. have suggested that AGE-BSA affects PLTs through serotonin receptor, the presence and even so the identity of receptor(s) for AGE proteins in PLTs has not been confirmed, yet. Herczenik et al.
postulaNe-(carboxymethyl)lysine) ated that PLT activation by amyloid-containing proteins is mediated throught 2 independent routes. It has been also revealed that RAGE is the receptor for $\beta$ amyloid $(A \beta)$ protein [26]. Since both AGE and $A \beta$ are the ligands for RAGE, it seems that these structures have similar domains to interact with this receptor [27]. We observed that incubation time of AGEHb affects secondary phase of ADP-induced aggregation remarkably. Obviously AGEHb20 was able to mimic Aspirin-like and AGEHb30 as Clopidrogrel-like anticoagulating agents, however. How AGEHb20 and 30 inhibit ADP-induced secondary phase of aggregation needs more investigation.

AGE-related fluorescence properties of the AGE-Hb were assessed in this study through maximum emission peak at $440 \mathrm{~nm}$. Fluorescence studies over a 30-day incubation period revealed an increase in the fluorescence intensity for the samples incubated with fructose invitro. ThT is used as a diagnostic dye for the amyloid fibrils due to its geometric fitness and therefore, binding to these structures $[28,29]$. Increase in fluorescence property of ThT upon interaction with AGE-Hb demonstrates the evolving of amyloid-like structure. Therefore, we monitored the amyloid fibrils formation as a result of alpha $(\alpha)$ to beta $(\beta)$ transition during glycation process as a function of incubation time through increase of ThT-associated fluorescence [30]. These data were also quantified CD spectroscopy in which an increase in $\beta$ content was resulted during the glycation process.

Since AGE-Hb affects PLT (ir-)responsiveness/ secretory phase, and it has amyloid-like structure, it seems that these domains are necessary for AGEs to affect PLTs (dys-)function. This assumption is consisted with the results from other groups who have already shown that AGEBSA induces PLT reactivity $[15,18]$ while some glycated structures like CML (Ne-(carboxymethyl)lysine) and pentosidine have no effect on these cells [16]. They have discussed their observation based on the presence of some essential epitopes in AGE-BSA. Moreover, Stanyer et al. [31] have shown an increase in PLT aggregation upon treatment with fibrilar form of $A \beta-40$, whereas aggregation is diminished when $\mathrm{A} \beta$-peptide has been treated in the presences of plasma lipoprotein due to wrapping of the amyloid structures and reducing its effect on PLTs. Regarding to these facts, the presence of RAGE or SR-like receptors on PLT surface for conducting AGE signaling seems to be necessary.

It has been shown that AGE structures affect PLT reactivity through the production of free radicals [32]. Oxidative stress plays an important role in AGE pathogenesis [33]. The main intracellular signaling pathways introduced by AGE structures might involve these molecules [34]. Hangaishi et al. [16] have indicated that interaction between AGE-BSA and PLTs produces intracellular superoxide onion [35]. They have also shown that in presence of antioxidants, the effect of AGE-BSA on PLT reactivity is significantly reduced. It is wellknown that $\mathrm{O}_{2}$ - triggers an increase in intracellular $\mathrm{Ca}^{+2}$ [36] which plays a central role in the regulation of PLT reactivity [37]. Therefore, it seems that AGE proteins cause a production in free radicals, and consequently an increase in inter- and intracellular calcium level in PLTs, which finally leads to PLT hyperactivity.

\section{Effects of NOS and ROS endproducts}

Nitric oxide acts as an essential molecule in the regulation of PLT function [38]. There are two NO synthase (NOS) insoforms in PLTs; iNOS and eNOS. The dominant form in normal individuals is eNOS, which is activated by $\mathrm{Ca}^{+2}$-calmodulin complex [4]. Here we are reporting the increase in NO production in PLTs via AGE-Hb 
treatment. Ferretti et al. have reported the same increase in NO level during incubation of PLT with glycated LDL. It seems that increase in intracellular $\mathrm{Ca}^{+2}$ during with the interaction between AGE and PLTs, probably through oxidative stress, activates eNOS, and consequently leads to production of NO [17]. It has been known that NO reduces Ca +2 concentration [39]; therefore, increase in NO production by PLT upon treatment with AGE may be a negative feedback mechanism for PLT hyperactivity. It has been revealed that $\mathrm{O}_{2^{-}}$reduces $\mathrm{NO}$ concentration by combining to it and form peroxynitrite (ONOO-). This combination has been speculated as a mechanism by which $\mathrm{O}_{2^{-}}$ diminishes the feedback effect of $\mathrm{NO}$ on $\mathrm{Ca}^{+2}$ and therefore, increases $\mathrm{Ca}^{+2}$ concentration [40,41]. Although, $\mathrm{ONOO}^{-}$is a pathogenic molecule in biological systems, its effect on PLT hyperactivity has not been clearly identified. In washed PLTs ONOO- induces aggregation; however, in PRP it acts as an inhibitor of PLT activity [42]. Therefore, it is not recognized that the formation of $\mathrm{ONOO}^{-}$is a mechanism for omitting $\mathrm{NO}$ by $\mathrm{O}_{2}$ - or diminishing $\mathrm{O}_{2}$ - effect by $\mathrm{NO}$.

Collectively, the present work provided here, put together with the results obtained by other groups, suggest that interaction between AGE-Hb and PLTs might occur through special receptors and secretory granula leading to production of free radicals and thereby an increase in inter- and intracellular $\mathrm{Ca}^{+2}$ concentration. Calcium might act in dual mode function to induce eNOS activity and production of $\mathrm{NO}$, which acts as a negative feedback for PLT function. Moreover, the combination between $\mathrm{O}_{2}$ - and $\mathrm{NO}$ produces $\mathrm{ONOO}^{-}$, which has a dual effect on PLT (ir-)responsiveness and reactivity. How fructose affects $\mathrm{Hb}$ structure and function and PLTs hyperreactivity in diabetes patients is still need more investigation. However, more studies are required to evaluate the definite mechanisms underlying these events.

\section{Acknowledgment}

This work was supported by grants from Iran National Science Foundation (INSF), grant number 8308 and Research Council of University of Tehran.

\section{Conflict of Interest}

There is no conflict of interest.

\section{References}

1. Sahud MA (1972) Clinical and biochemical characteristics of a primary platelet disorder. Acta Univ Carol Med Monogr 53: 317-324.

2. Sobol AB, Watala C (2000) The role of platelets in diabetes-related vascular complications. Diabetes Res Clin Pract 50: 1-16.

3. Vinik AI, Erbas T, Park TS, Nolan R, Pittenger GL (2001) Platelet dysfunction in type 2 diabetes. Diabetes Care 24: 1476-1485.

4. Mazzanti L, Mutus B (1997) Diabetes-induced alterations in platelet metabolism. Clin Biochem 30: 509-515.

5. Iuliano L, Colavita AR, Leo R, Praticò D, Violi F (1997) Oxygen free radicals and platelet activation. Free Radic Biol Med 22: 999-1006.

6. Yim MB, Yim HS, Lee C, Kang SO, Chock PB (2001) Protein glycation: creation of catalytic sites for free radical generation. Ann N Y Acad Sci 928: 48-53.

7. Singh R, Barden A, Mori T, Beilin L (2001) Advanced glycation endproducts: a review. Diabetologia 44: 129-146.

8. Luthra M, Balasubramanian D (1993) Nonenzymatic glycation alters protein structure and stability. A study of two eye lens crystallins. J Biol Chem 268: 18119-18127.

9. Vlassara H, Fuh H, Makita Z, Krungkrai S, Cerami A, et al. (1992) Exogenous advanced glycosylation end products induce complex vascular dysfunction in normal animals: a model for diabetic and aging complications. Proc Natl Acad Sci USA 89: 12043-1247.

10. Ahmed N, Thornalley PJ (2007) Advanced glycation endproducts: what is their relevance to diabetic complications? Diabetes Obes Metab 9: 233-245.

11. Frolov A, Hoffmann R (2008) Separation of Amadori peptides from their unmodified analogs by ion-pairing RP-HPLC with heptafluorobutyric acid as ion-pair reagent. Anal Bioanal Chem 392: 1209-1214.

12. Khazaei MR, Habibi-Rezaei M, Karimzadeh F, Moosavi-Movahedi AA, Sarrafnejhad AA, et al. (2008) Microglial cell death induced by glycated bovine serum albumin: nitric oxide involvement. J Biochem 144: 197-206.

13. Sampietro T, Lenzi S, Cecchetti $\mathrm{P}$, Giampietro $\mathrm{O}$, Cruschelli $\mathrm{L}$, et al. (1986) Nonenzymatic glycation of human platelet membrane proteins in vitro and in vivo. Clin Chem 32: 1328-1331.

14. Muruganandam A, Romsa GJ, Thibert RJ, Cheung RM, Draisey TF, et al. (1993) Glycated calmodulin from platelets as an index of glycemic control. Clin Chem 39: 815-819.

15. Herczenik E, Bouma B, Korporaal SJ, Strangi R, Zeng Q, et al. (2007) Activation of human platelets by misfolded proteins. Arterioscler Thromb Vasc Biol 27: 1657-1665.

16. Hangaishi M, Taguchi J, Miyata T, Ikari Y, Togo M, et al. (1998) Increased aggregation of human platelets produced by advanced glycation end products in vitro. Biochem Biophys Res Commun 248: 285-292.

17. Ferretti G, Rabini RA, Bacchetti T, Vignini A, Salvolini E, et al. (2002) Glycated low density lipoproteins modify platelet properties: a compositional and functional study. J Clin Endocrinol Metab 87: 2180-2184.

18. Ahmad W, Li L, Deng Y (2008) Identification of AGE-precursors and AGE formation in glycation-induced BSA peptides. BMB Rep 41: 516-522.

19. Makita Z, Vlassara H, Rayfield E, Cartwright K, Friedman E, et al. (1992) Hemoglobin-AGE: a circulating marker of advanced glycosylation. Science 258: 651-653.

20. Shapiro BP, Owan TE, Mohammed SF, Meyer DM, Mills LD, et al. (2008) Advanced glycation end products accumulate in vascular smooth muscle and modify vascular but not ventricular properties in elderly hypertensive canines. Circulation 118: 1002-1010.

21. Bakhti M, Habibi-Rezaei M, Moosavi-Movahedi AA, Khazaei MR (2007) Consequential alterations in haemoglobin structure upon glycation with fructose: prevention by acetylsalicylic acid. J Biochem 141: 827-833.

22. Bradford MM (1976) A rapid and sensitive method for the quantitation of microgram quantities of protein utilizing the principle of protein-dye binding. Anal Biochem 72: 248-254.

23. Böhm G, Muhr R, Jaenicke R (1992) Quantitative analysis of protein far UV circular dichroism spectra by neural networks. Protein Eng 5: 191-195.

24. Ferroni P, Basili S, Falco A, Davì G (2004) Platelet activation in type 2 diabetes mellitus. J Thromb Haemost 2: 1282-1291.

25. Hofmann MA, Drury S, Fu C, Qu W, Taguchi A, et al. (1999) RAGE mediates a novel proinflammatory axis: a central cell surface receptor for S100/calgranulin polypeptides. Cell 97: 889-901.

26. Yan SD, Chen X, Fu J, Chen M, Zhu H, et al. (1996) RAGE and amyloidbeta peptide neurotoxicity in Alzheimer's disease. Nature 382: 685-691.

27. Obrenovich ME, Monnier VM (2004) Glycation stimulates amyloid formation. Sci Aging Knowledge Environ 2004: pe3.

28. Krebs MR, Bromley EH, Donald AM (2005) The binding of thioflavin-T to amyloid fibrils: localisation and implications. J Struct Biol 149: 30-37.

29. Khurana R, Coleman C, Ionescu-Zanetti C, Carter SA, Krishna V, et al. (2005) Mechanism of thioflavin T binding to amyloid fibrils. J Struct Biol 151: 229-238.

30. Vasconcelos E, Figueiredo AC, Seghatchian J (2003) Quality of platelet concentrates derived by platelet rich plasma, buffy coat and Apheresis. Transfus Apher Sci 29: 13-16. 
Citation: $\quad$ Asadifar M, Bakhti M, Habibi-Rezaei M, Moosavi-Movahedi AA, Tabatabi MR, et al. (2015) Platelet Aggregation Increased by Advanced Glycated Hemoglobin. J Blood Disord Transfus 6: 293. doi:10.4172/2155-9864.1000293

Page 6 of 6

31. Stanyer L, Betteridge DJ, Smith CC (2004) Exaggerated polymerisation of beta-amyloid 40 stimulated by plasma lipoproteins results in fibrillar Abeta preparations that are ineffective in promoting ADP-induced platelet aggregation. Biochim Biophys Acta 1674: 305-311.

32. Mazzanti L, Nanetti L, Vignini A, Rabini RA, Grechi G, et al. (2004) Gestational diabetes affects platelet behaviour through modified oxidative radical metabolism. Diabet.Med 21: 68-72.

33. Lal S, Chithra P, Chandrakasan G (1996) The possible relevance of autoxidative glycosylation in glucose mediated alterations of proteins: an in vitro study on myofibrillar proteins. Mol Cell Biochem 154: 95-100.

34. Ahmed N, Babaei-Jadidi R, Howell SK, Thornalley PJ, Beisswenger PJ (2005) Glycated and oxidized protein degradation products are indicators of fasting and postprandial hyperglycemia in diabetes. Diabetes Care 28: 2465-2471.

35. Praticò D, Iuliano L, Alessandri C, Camastra C, Violi F (1993) Polymorphonuclear leukocyte-derived O2-reactive species activate primed platelets in human whole blood. Am J Physiol 264: H1582-1587.

36. Masumoto N, Tasaka K, Miyake A, Tanizawa O (1990) Superoxide anion increases intracellular free calcium in human myometrial cells. J Biol Chem 265: 22533-22536.
37. Sage SO, Sargeant P, Heemskerk JW, Mahaut-Smith MP (1993) Calcium influx mechanisms and signal organisation in human platelets. Adv Exp Med Biol 344: 69-82.

38. Gkaliagkousi E, Ritter J, Ferro A (2007) Platelet-derived nitric oxide signaling and regulation. Circ Res 101: 654-662.

39. Trepakova ES, Cohen RA, Bolotina VM (1999) Nitric oxide inhibits capacitative cation influx in human platelets by promoting sarcoplasmic/ endoplasmic reticulum $\mathrm{Ca} 2+-\mathrm{ATPase}$-dependent refilling of $\mathrm{Ca} 2+$ stores. Circ.Res 84: 201-209.

40. Jansen MA, Badlou BA, van Echteld CJ, Ruigrok TJ (2000) Cytosolic $\mathrm{Ca} 2+$ concentration during $\mathrm{Ca} 2+$ depletion of isolated rat hearts. Mol Cell Biochem 203: 169-175.

41. Krotz F, Riexinger T, Buerkle MA, Nithipatikom K, Gloe T, et al. (2004) Membrane-potential-dependent inhibition of platelet adhesion to endothelial cells by epoxyeicosatrienoic acids. Arterioscler.Thromb.Vasc.Biol 24: 595-600.

42. Moro MA, Darley-Usmar VM, Goodwin DA, Read NG, Zamora-Pino R, et al. (1994) Paradoxical fate and biological action of peroxynitrite on human platelets. Proc Natl Acad Sci U S A 91: 6702-6706. 